\title{
Numerical Treatment of Fixed Point Applied to the Nonlinear Fredholm Integral Equation
}

\section{I. Berenguer, M. V. Fernández Muñoz, A. I. Garralda Guillem, and M. Ruiz Galán}

Departamento de Matemática Aplicada, Universidad de Granada, E.U. Arquitectura Técnica, c/ Severo

Ochoa s/n, 18071 Granada, Spain

Correspondence should be addressed to M. Ruiz Galán, mruizg@ugr.es

Received 15 May 2009; Accepted 8 July 2009

Recommended by Massimo Furi

The authors present a method of numerical approximation of the fixed point of an operator, specifically the integral one associated with a nonlinear Fredholm integral equation, that uses strongly the properties of a classical Schauder basis in the Banach space $C([a, b] \times[a, b])$.

Copyright (c) 2009 M. I. Berenguer et al. This is an open access article distributed under the Creative Commons Attribution License, which permits unrestricted use, distribution, and reproduction in any medium, provided the original work is properly cited.

\section{Introduction}

Let us consider the nonlinear Fredholm integral equation of the second kind

$$
f(x)=\lambda u(x)-\int_{a}^{b} k(x, y, u(y)) d y
$$

where $\lambda \in \mathbb{R} \backslash\{0\}$ and $f:[a, b] \rightarrow \mathbb{R}$ and $k:[a, b] \times[a, b] \times \mathbb{R} \rightarrow \mathbb{R}$ are continuous functions. By defining in the Banach space $C([a, b])$ of those continuous and real-valued functions defined on $[a, b]$ (usual sup norm) the integral operator $T: C([a, b]) \rightarrow C([a, b])$ as

$$
T(v)(x)=\frac{1}{\lambda} f(x)+\frac{1}{\lambda} \int_{a}^{b} k(x, y, v(y)) d y, \quad(x \in[a, b], v \in C([a, b])),
$$

then the Banach fixed point, theorem guarantees that, under certain assumptions, $T$ has a unique fixed point; that is, the Fredholm integral equation has exactly one solution. Indeed, assume in addition that $k$ is a Lipschitz function at its third variable with Lipschitz constant 
$M>0$ and that $|\lambda|>M(b-a)$, then the operator $T$ is contractive with contraction number $\alpha:=M(b-a) /|\lambda|$, and thus $T$ has a unique fixed point $\varphi$. Moreover, $\varphi=\lim _{n \rightarrow \infty} T^{n}\left(\varphi_{0}\right)$, where $\varphi_{0}$ is any continuous function on $[a, b]$. Since in general it is not possible to calculate explicitly from a $\varphi_{0}$ the sequence of functions $\left\{T^{n}\left(\varphi_{0}\right)\right\}_{n \in \mathbb{N}}$, we define in this work a new sequence of functions, denoted by $\left\{\varphi_{h}\right\}_{h \in \mathbb{N}}$, obtained recursively making use of certain Schauder basis in $C([a, b] \times[a, b])$ (Banach space of those continuous real-valued functions on $[a, b] \times[a, b]$ endowed with its usual sup norm). More concretely, we get $\varphi_{h+1}$ from $\varphi_{h}$, approximating $T\left(\varphi_{h}\right)$ by means of the sequence of projections of such Schauder basis.

\section{Numerical Approximation of the Solution}

We start by recalling certain aspects about some well-known Schauder bases in the Banach spaces $C([a, b])$ and $C([a, b] \times[a, b])$.

Let us consider the usual Schauder basis $\left\{s_{n}\right\}_{n \geq 0}$ in $C([a, b])$, that is, for a dense sequence of distinct points $\left\{t_{n}^{\prime}\right\}_{n \geq 0}$ in $[a, b]$ with $t_{0}^{\prime}=a$ and $t_{1}^{\prime}=b$, we define

$$
s_{0}\left(t^{\prime}\right):=1, \quad\left(t^{\prime} \in[a, b]\right),
$$

and for $n>0 ; s_{n}$ is the piecewise linear and continuous function with nodes $\left\{t_{0}^{\prime}, t_{1}^{\prime}, \ldots, t_{n}^{\prime}\right\}$ satisfying for all $i<n, s_{n}\left(t_{i}^{\prime}\right)=0$ and $s_{n}\left(t_{n}^{\prime}\right)=1$. From this Schauder basis we define the usual Schauder basis $\left\{S_{n}\right\}_{n \geq 0}$ for $C([a, b] \times[a, b])$. We consider the bijective mapping $\tau: \mathbb{N} \rightarrow \mathbb{N} \times \mathbb{N}$, ([ ] denotes integer part) given by

$$
\tau(n)= \begin{cases}(\sqrt{n}, \sqrt{n}), & \text { if } \sqrt{n}=[\sqrt{n}], \\ \left(n-[\sqrt{n}]^{2},[\sqrt{n}]+1\right), & \text { if } 0<n-[\sqrt{n}]^{2} \leq[\sqrt{n}], \\ \left([\sqrt{n}]+1, n-[\sqrt{n}]^{2}-[\sqrt{n}]\right), & \text { if }[\sqrt{n}]<n-[\sqrt{n}]^{2},\end{cases}
$$

and take, for each $n \in \mathbb{N}$ with $\tau(n)=(i, j)$,

$$
S_{n}(x, y):=s_{i}(x) s_{j}(y), \quad(x, y \in[a, b])
$$

The sequence $\left\{S_{n}\right\}_{n \geq 0}$ is the usual Schauder basis in $C([a, b] \times[a, b])$ (see [1]). We will denote by $\left\{S_{n}^{*}\right\}_{n \geq 0}$ and $\left\{Q_{n}\right\}_{n \geq 0}$, respectively, the sequences of biorthogonal functionals and projections associated with such basis, that is, given $\psi \in C([a, b] \times[a, b])$, the (continuous) functionals $S_{n}^{*}: C([a, b] \times[a, b]) \rightarrow \mathbb{R}$ verify

$$
\psi=\sum_{n=0}^{\infty} S_{n}^{*}(\psi) S_{n}
$$

and the (continuous) projections are defined by

$$
Q_{n}=\sum_{i=0}^{n} S_{i}^{*}(\psi) S_{i}
$$


Let us now introduce some notational conventions. For each $n$ the definition of projection $Q_{n^{2}}$ just needs the first $n+1$ points of the sequence $\left\{t_{n}^{\prime}\right\}_{n \geq 0}$, ordered in an increasing way that will be denoted by $\Delta_{n}:=\left\{t_{0}=a, t_{1}, \ldots, t_{n}=b\right\}$, and in addition we will write $p:=\max _{0 \leq i \leq n-1}\left(t_{i+1}-t_{i}\right)$.

We now describe idea of the numerical method proposed. The beginning point is the operator formulation of the integral Fredholm equation; from an initial function $\varphi_{0} \in$ $C([a, b])$, and since in general we cannot calculate explicitly $T\left(\varphi_{0}\right)$, we approximate this function in the following way: let

$$
\psi_{0}(x, y):=k\left(x, y, \varphi_{0}(y)\right), \quad(x, y \in[a, b])
$$

so

$$
T\left(\varphi_{0}\right)(x)=\frac{1}{\lambda} f(x)+\frac{1}{\lambda} \int_{a}^{b} \psi_{0}(x, y) d y \approx \frac{1}{\lambda} f(x)+\frac{1}{\lambda} \int_{a}^{b} Q_{m_{0}}\left(\psi_{0}\right)(x, y) d y,
$$

where $m_{0}$ is an adequate integer. We denote the last function by $\varphi_{1}$ and repeat the same construction. Then we define recursively for each $x, y \in[a, b], h \geq 1$ and $m_{h-1} \in \mathbb{N}$,

$$
\begin{aligned}
\psi_{h-1}(x, y) & :=k\left(x, y, \varphi_{h-1}(y)\right), \\
\varphi_{h}(x) & :=\frac{1}{\lambda} f(x)+\frac{1}{\lambda} \int_{a}^{b} Q_{m_{h-1}}\left(\psi_{h-1}\right)(x, y) d y .
\end{aligned}
$$

Now we state some technical results in order to study the error $\left\|\varphi-\varphi_{h}\right\|_{\infty}$. In the first of them we give a bound for the distance between a continuous function and its projections. It is not difficult to prove it as a consequence of the Mean Value Theorem and the following interpolation property satisfied by the sequence of projections $\left\{Q_{n}\right\}_{n \geq 0}$ (see [1]): whenever $n, i, j \in \mathbb{N} \cup\{0\}$ and $\tau^{-1}(i, j)<n^{2}$, then

$$
\forall t_{i}, t_{j} \in \Delta_{n}, \quad Q_{n^{2}}(\psi)\left(t_{i}, t_{j}\right)=\psi\left(t_{i}, t_{j}\right)
$$

Lemma 2.1. Let $\psi \in C^{1}([a, b] \times[a, b])$, let $L=\max \left\{\|\partial \psi / \partial x\|_{\infty},\|\partial \psi / \partial y\|_{\infty}\right\}$, and let $\left\{Q_{n}\right\}_{n \geq 0}$ be the sequence of projections associated with the basis $\left\{S_{n}\right\}_{n \geq 1}$, then it holds that

$$
\left\|\psi-Q_{n^{2}}(\psi)\right\|_{\infty} \leq 4 L p
$$

Let us introduce some notation, useful in what follows: given $h \geq 1$, we write

$$
\begin{aligned}
L_{h-1} & :=\max \left\{\left\|\frac{\partial \psi_{h-1}}{\partial x}\right\|_{\infty},\left\|\frac{\partial \psi_{h-1}}{\partial y}\right\|_{\infty}\right\} \\
p_{h} & :=\max _{0 \leq i \leq n_{h-1}}\left(t_{i+1}-t_{i}\right), \quad n_{h} \geq 2, t_{i} \in \Delta_{n_{h}} .
\end{aligned}
$$


Lemma 2.2. Suppose that $\varphi_{0} \in C^{1}([a, b]), k \in C^{1}([a, b] \times[a, b] \times \mathbb{R}), \lambda \neq 0$, and $T: C([a, b]) \rightarrow$ $C([a, b])$ is the operator given by

$$
T(v)(x)=\frac{1}{\lambda} f(x)+\frac{1}{\lambda} \int_{a}^{b} k(x, y, v(y)) d y, \quad(x \in[a, b], v \in C([a, b])) .
$$

Then, maintaining the preceding notation, we have that for all $h \geq 1$,

$$
\left\|T\left(\varphi_{h-1}\right)-\varphi_{h}\right\|_{\infty} \leq \frac{b-a}{|\lambda|} 4 L_{h-1} p_{h}
$$

Proof. Since $\psi_{h-1} \in C([a, b] \times[a, b])$ and $\left\{S_{n}\right\}_{n \geq 0}$ is a Schauder basis for the Banach space $C([a, b] \times[a, b])$, then

$$
\psi_{h-1}=\sum_{n=0}^{\infty} S_{n}^{*}\left(\psi_{h-1}\right) S_{n}
$$

On the other hand, taking into account the definition of $\varphi_{h}$, we have that

$$
\begin{aligned}
\left|T\left(\varphi_{h-1}\right)(x)-\varphi_{h}(x)\right| & =\left|\frac{1}{\lambda} \int_{a}^{b}\left(\sum_{n=0}^{\infty} S_{n}^{*}\left(\psi_{h-1}\right) S_{n}(x, y)-\sum_{n=0}^{m_{h-1}} S_{n}^{*}\left(\psi_{h-1}\right) S_{n}(x, y)\right) d y\right| \\
& =\left|\frac{1}{\lambda} \int_{a}^{b}\left(\psi_{h-1}(x, y)-Q_{m_{h-1}^{2}}\left(\psi_{h-1}\right)(x, y)\right) d y\right| .
\end{aligned}
$$

Finally, in view of Lemma 2.1 we arrive at

$$
\left\|T\left(\varphi_{h-1}\right)-\varphi_{h}\right\|_{\infty} \leq \frac{(b-a)}{|\lambda|} 4 L_{h-1} p_{h}
$$

Finally we arrive at the following estimation of the error.

Theorem 2.3. Assume that $\lambda \neq 0, \varphi_{0} \in C^{1}([a, b]), k \in C^{1}([a, b] \times[a, b] \times \mathbb{R})$ is a lipschitzian function at its third variable with Lipschitz constant $M$ with $|\lambda|>M(b-a)$ and that $\varphi$ is the unique fixed point of the integral operator $T: C([a, b]) \rightarrow C([a, b])$ defined by

$$
T(v)(x)=\frac{1}{\lambda} f(x)+\frac{1}{\lambda} \int_{a}^{b} k(x, y, v(y)) d y, \quad(x \in[a, b], v \in C([a, b])) .
$$

Suppose in addition that $h \geq 1$ and that $\varepsilon_{1}, \ldots, \varepsilon_{h}>0$ satisfy

$$
1 \leq j \leq h \Longrightarrow \frac{(b-a)}{|\lambda|} 4 L_{j-1} p_{j}<\varepsilon_{j}
$$


Fixed Point Theory and Applications

Then, with the previous notation, it is satisfied that

$$
\left\|\varphi-\varphi_{h}\right\|_{\infty} \leq\left\|T\left(\varphi_{0}\right)-\varphi_{0}\right\|_{\infty} \frac{\alpha^{h}}{1-\alpha}+\sum_{j=1}^{h} \alpha^{h-j} \varepsilon_{j},
$$

where $\alpha=M(b-a) /|\lambda|$.

Proof. We begin with the triangular inequality

$$
\left\|\varphi-\varphi_{h}\right\|_{\infty} \leq\left\|\varphi-T^{h}\left(\varphi_{0}\right)\right\|_{\infty}+\left\|T^{h}\left(\varphi_{0}\right)-\varphi_{h}\right\|_{\infty} .
$$

In order to obtain a bound for the first right-hand side term we observe that operator $T$ is contractive, with contraction constant $\alpha \in(0,1)$. Hence the Banach fixed point Theorem gives that

$$
\begin{gathered}
\varphi=\lim _{m \rightarrow \infty} T^{m}\left(\varphi_{0}\right), \\
\left\|\varphi-T^{h}\left(\varphi_{0}\right)\right\|_{\infty} \leq\left\|T\left(\varphi_{0}\right)-\varphi_{0}\right\|_{\infty} \frac{\alpha^{h}}{1-\alpha} .
\end{gathered}
$$

For deducing a bound for the second right-hand side term of (2.20), we use Lemma 2.2 and the assumption $j=1, \ldots, h \Rightarrow((b-a) /|\lambda|) 4 L_{j-1} p_{j}<\varepsilon_{j}$ in the following chain of inequalities:

$$
\begin{aligned}
\left\|T^{h}\left(\varphi_{0}\right)-\varphi_{h}\right\|_{\infty} & \leq\left\|T^{h}\left(\varphi_{0}\right)-T\left(\varphi_{h-1}\right)\right\|_{\infty}+\left\|T\left(\varphi_{h-1}\right)-\varphi_{h}\right\|_{\infty} \\
& \leq\left\|T^{h}\left(\varphi_{0}\right)-T\left(\varphi_{h-1}\right)\right\|_{\infty}+\frac{(b-a)}{|\lambda|} 4 L_{h-1} p_{h} \\
& \leq \alpha\left\|T^{h-1}\left(\varphi_{0}\right)-\varphi_{h-1}\right\|_{\infty}+\varepsilon_{h} \\
& \leq \alpha\left(\left\|T^{h-1}\left(\varphi_{0}\right)-T\left(\varphi_{h-2}\right)\right\|_{\infty}+\left\|T\left(\varphi_{h-2}\right)-\varphi_{h-1}\right\|_{\infty}\right)+\varepsilon_{h} \\
& \leq \alpha\left\|T\left(T^{h-2}\left(\varphi_{0}\right)\right)-T\left(\varphi_{h-2}\right)\right\|_{\infty}+\alpha \varepsilon_{h-1}+\varepsilon_{h} \\
& \leq \alpha^{2}\left\|T^{h-2}\left(\varphi_{0}\right)-\varphi_{h-2}\right\|_{\infty}+\alpha \varepsilon_{h-1}+\varepsilon_{h} \\
& \leq \alpha^{2}\left(\left\|T^{h-2}\left(\varphi_{0}\right)-T\left(\varphi_{h-3}\right)\right\|_{\infty}+\left\|T\left(\varphi_{h-3}\right)-\varphi_{h-2}\right\|_{\infty}\right)+\alpha \varepsilon_{h-1}+\varepsilon_{h} \\
& \leq \cdots \\
& \leq \alpha^{h-1}\left\|T\left(\varphi_{0}\right)-\varphi_{1}\right\|_{\infty}+\alpha^{h-2} \varepsilon_{2}+\cdots+\alpha^{2} \varepsilon_{h-2}+\alpha \varepsilon_{h-1}+\varepsilon_{h} .
\end{aligned}
$$


Once again, in view of Lemma 2.2 it follows that

$$
\left\|T^{h} \varphi_{0}-\varphi_{h}\right\|_{\infty} \leq \sum_{j=1}^{h} \alpha^{h-j} \varepsilon_{j}
$$

Therefore, inequalities (2.20), (2.22), and (2.24) allow us to conclude that

$$
\left\|\varphi-\varphi_{h}\right\|_{\infty}<\left\|T\left(\varphi_{0}\right)-\varphi_{0}\right\|_{\infty} \frac{\alpha^{h}}{1-\alpha}+\sum_{j=1}^{h} \alpha^{h-j} \varepsilon_{j},
$$

as announced.

Remarks 2.4. (1) The linear case was previously stated in [2]. For a general overview of the classical methods, see $[3,4]$.

(2) The use of Schauder bases in the numerical study of integral and differential equations has been previously considered in [5-7] or [8].

(3) For other approximating methods in Hilbert or Banach spaces, we refer to $[9,10]$.

\section{Numerical Examples}

We finally illustrate the numerical method proposed above by means of the two following examples. In both of them we choose the dense subset of $[0,1]$

$$
\left\{0,1, \frac{1}{2}, \frac{1}{4}, \frac{3}{4}, \ldots, \frac{1}{2^{k}}, \frac{3}{2^{k}}, \ldots, \frac{2^{k}-1}{2^{k}}, \ldots\right\}
$$

to construct the Schauder bases in $C([0,1])$ and $C([0,1] \times[0,1])$. To define the sequence of approximating functions $\left\{\varphi_{h}\right\}_{h \in \mathbb{N}}$ we have taken an initial function $\varphi_{0} \in C^{1}([0,1])$ and for all $h \geq 1, m_{h}=n^{2}$ with different values of $n$ of the form $n=2^{k}+1$, with $k \in \mathbb{N}$. For such a choice, the value $p_{h}$ appearing in Lemma 2.2 is $p_{h}=1 / 2^{k}$, for all $h \in \mathbb{N}$.

Example 3.1. Let us consider the nonlinear Fredholm integral equation of the second kind in $[0,1]$ :

$$
2 x+\frac{(2-\pi) x^{2}}{8}=2 u(x)-\int_{0}^{1}\left(\frac{x^{2} y}{2} \arctan (u(y))\right) d y,
$$

whose analytical solution is the function $\varphi(x)=x$. In Table 1 we exhibit the absolute errors committed in nine points $x_{i}$ in $[0,1]$ when we approximate the exact solution $\varphi$ by the iteration $\varphi_{m}$, by considering different values of $n(n=9,17,33)$. 
Table 1: Example 3.1.

\begin{tabular}{lccc}
\hline$x_{i}$ & $n=9, m=3$ & $n=17, m=3$ & $n=33, m=3$ \\
\hline 0. & 0. & 0. & 0. \\
0.125 & $6.41821 \times 10^{-6}$ & $1.31795 \times 10^{-6}$ & $4.35379 \times 10^{-8}$ \\
0.25 & $2.56728 \times 10^{-5}$ & $5.27181 \times 10^{-6}$ & $1.74152 \times 10^{-7}$ \\
0.375 & $5.77639 \times 10^{-5}$ & $1.18616 \times 10^{-5}$ & $3.91841 \times 10^{-7}$ \\
0.5 & $1.02691 \times 10^{-4}$ & $2.10873 \times 10^{-5}$ & $6.96607 \times 10^{-7}$ \\
0.625 & $1.60455 \times 10^{-4}$ & $3.29488 \times 10^{-5}$ & $1.08845 \times 10^{-6}$ \\
0.75 & $2.31055 \times 10^{-4}$ & $4.74463 \times 10^{-5}$ & $1.56737 \times 10^{-6}$ \\
0.875 & $3.14492 \times 10^{-4}$ & $6.45797 \times 10^{-5}$ & $2.13336 \times 10^{-6}$ \\
1. & $4.10765 \times 10^{-4}$ & $8.4349 \times 10^{-5}$ & $2.78643 \times 10^{-6}$ \\
\hline
\end{tabular}

Table 2: Example 3.2.

\begin{tabular}{lcc}
\hline$p$ & $E_{p}$ & $F_{p}$ \\
\hline 8 & $3.27 \times 10^{-4}$ & $1.86 \times 10^{-4}$ \\
16 & $8.18 \times 10^{-5}$ & $4.61 \times 10^{-5}$ \\
32 & $2.04 \times 10^{-5}$ & $1.12 \times 10^{-5}$ \\
64 & $5.11 \times 10^{-6}$ & $2.72 \times 10^{-6}$ \\
\hline
\end{tabular}

Example 3.2. Now we consider the following Fredholm integral equation appearing in [5, Example (11.2.1)]:

$$
f(x)=5 u(x)-\int_{0}^{1} e^{x y} u(y) d y
$$

where $f$ is defined in such a way that $u(x)=e^{-x} \cos x$ is the exact solution. We denote by $u_{p}$ the approximation of the exact solution given by the collocation method and by $E_{p}$ the error:

$$
E_{p}:=\max _{1 \leq j \leq p+1}\left|u\left(x_{j}\right)-u_{n}\left(x_{j}\right)\right|
$$

where $\left\{x_{j}\right\}_{j=1}^{n+1}$ are the nodes of the collocation method. Now write $F_{p}$ for the error

$$
F_{p}:=\max _{1 \leq j \leq p+1}\left|u\left(x_{j}\right)-\varphi_{m}\left(x_{j}\right)\right|
$$

with $\varphi_{m}$ being the approximation obtained with our method, with $m_{h}=(p+1)^{2}$ for $h=$ $1, \ldots, m$ and choosing $m$ in such a way that

$$
\frac{F_{m}}{F_{m+1}}<1+10^{-2}
$$

In Table 2 we show the errors for both methods. 
Remark 3.3. Although the errors obtained in the preceding example by our algorithm are similar to those derived from the collocation method, the computational cost is quite different: in order to apply the collocation method we need to solve high-order linear systems of algebraical equations, but for our method we just calculate linear combinations of scalar obtained by evaluating adequate functions. Indeed, the sequence of biorthogonal functionals $\left\{S_{n}^{*}\right\}_{n \in \mathbb{N}}$ satisfies the following easy property (see [1]): for all $v \in C\left([0,1]^{2}\right)$,

$$
S_{1}^{*}(v)=v\left(t_{0}, t_{0}\right)
$$

while for all $n \geq 1$, if $\tau(n)=(i, j)$,

$$
S_{n}^{*}(v)=v\left(t_{i}, t_{j}\right)-\sum_{k=0}^{n-1} S_{k}^{*}(v) B_{k}\left(t_{i}, t_{j}\right) .
$$

Obviously, this easy way of determining the biorthogonal functionals and consequently the approximating functions $\varphi_{h}$ (integrals of a piecewise linear function) is equally valid in the general nonlinear case.

\section{Acknowledgments}

This research partially supported by M.E.C. (Spain) and FEDER project no. MTM2006-12533 and by Junta de Andalucía Grant FQM359.

\section{References}

[1] Z. Semadeni, Schauder Bases in Banach Spaces of Continuous Functions, vol. 918 of Lecture Notes in Mathematics, Springer, Berlin, Germany, 1982.

[2] M. Domingo Montesinos, A. I. Garralda Guillem, and M. Ruiz Galán, "Fredholm integral equations and Schauder bases," in VIII Journées Zaragoza-Pau de Mathématiques Appliquées et de Statistiques, vol. 31 of Monografías del Seminario Matemático García de Galdeano, pp. 121-128, Zaragoza University Press, Zaragoza, Spain, 2004.

[3] M. A. Golberg, Numerical Solution of Integral Equations, vol. 42 of Mathematical Concepts and Methods in Science and Engineering, Plenum Press, New York, NY, USA, 1990.

[4] K. E. Atkinson and W. Han, Theoretical Numerical Analysis, Springer, New York, NY, USA, 2nd edition, 2005.

[5] M. I. Berenguer, M. A. Fortes, A. I. Garralda Guillem, and M. Ruiz Galán, "Linear Volterra integrodifferential equation and Schauder bases," Applied Mathematics and Computation, vol. 159, no. 2, pp. 495-507, 2004.

[6] D. Gámez, A. I. Garralda Guillem, and M. Ruiz Galán, “Nonlinear initial-value problems and Schauder bases," Nonlinear Analysis: Theory, Methods \& Applications, vol. 63, no. 1, pp. 97-105, 2005.

[7] D. Gámez, A. I. Garralda Guillem, and M. Ruiz Galán, “High-order nonlinear initial-value problems countably determined," Journal of Computational and Applied Mathematics, vol. 228, no. 1, pp. 77-82, 2009.

[8] A. Palomares and M. Ruiz Galán, "Isomorphisms, Schauder bases in Banach spaces, and numerical solution of integral and differential equations," Numerical Functional Analysis and Optimization, vol. 26, no. 1, pp. 129-137, 2005.

[9] Z. Gu and Y. Li, "Approximation methods for common fixed points of mean nonexpansive mapping in Banach spaces," Fixed Point Theory and Applications, vol. 2008, Article ID 471532, 7 pages, 2008.

[10] Y. Yao and R. Chen, "Iterative algorithm for approximating solutions of maximal monotone operators in Hilbert spaces," Fixed Point Theory and Applications, vol. 2007, Article ID 32870, 8 pages, 2007. 